\title{
Co-phasing primary mirror segments of an optical space telescope using a long stroke Zernike WFS
}

\author{
Kate Jackson ${ }^{a}$, J. Kent Wallace ${ }^{b}$, Sergio Pellegrino ${ }^{a}$ \\ ${ }^{a}$ Division of Aerospace Engineering, California Institute of Technology, 1200 E. \\ California Blvd., Pasadena CA 91125, USA; \\ ${ }^{b}$ Jet Propulsion Laboratory/California Institute of Technology, 4800 Oak Grove \\ Dr., Pasadena CA 91109
}

\begin{abstract}
Static Zernike phase-contrast plates have been used extensively in microscopy for half a century and, more recently, in optical telescopes for wavefront sensing. A dynamic Zernike wavefront sensor (WFS) with four phase shifts, for reducing error due to spurious light and eliminating other asynchronous noise, has been proposed for use in adaptive optics. Here, we propose adapting this method for co-phasing the primary mirror of a segmented space telescope. In order to extend the dynamic range of the WFS, which has a maximum range of $+/-\lambda / 2$, a phasecontrast plate with multiple steps, both positive and negative, has been developed such that errors as large as $+/-10 \lambda$ can be sensed. The manufacturing tolerances have been incorporated into simulations, which demonstrate that performance impacts are minimal. We show that the addition of this small optical plate along with a high precision linear translation stage at the prime focus of a telescope and pupil viewing capability can provide extremely accurate segment phasing with a simple white-light fringe fitting algorithm and a closed-loop controller. The original focal-plane geometry of a centro-symmetric phase shifting element is replaced with a much less constrained shape, such as a slot. Also, a dedicated pupil imager is not strictly required; an existing pupil sampler such as a Shack-Hartmann (SH) WFS can be used just as effectively, allowing simultaneous detection of wavefront errors using both intensity and spot positions on the SH-WFS. This could lead to an efficient synergy between Zernike and SH-WFS, enabling segment phasing in conjunction with high-dynamic range sensing.
\end{abstract}

\section{INTRODUCTION}

Modern large aperture optical/near-IR telescopes, both ground and space-based, have reached sizes such that segmented primary mirrors are required to move forward. For ground-based telescopes, the primary mirror diameters have already surpassed the practical limit for monolithic mirrors with the latest generation of 10m-class facilities, and all three next generation Extremely Large Telescopes (Thirty Metre Telescope, Giant Magellan Telescope, and European Extremely Large Telescope) will expand on the segmented primary concept in various ways to reach their target diameters of $30 \mathrm{~m}, 25 \mathrm{~m}$, and $42 \mathrm{~m}$ respectively. ${ }^{1-3}$ For space-based telescopes, primary mirror diameters are limited by other factors such as launch weight and rocket faring size. For these reasons, the James Webb Space Telescope (JWST) project will implement a foldable segmented primary mirror and support structure which will deploy after launch. ${ }^{4}$ The future of space-based facilities will also look to expand on the segmented concept with the goal of attaining 20-30m apertures. ${ }^{5}$

Further author information: (Send correspondence to K. Jackson) K. Jackson: E-mail: kathrynj@caltech.edu, Telephone: 16263954452

Space Telescopes and Instrumentation 2016: Optical, Infrared, and Millimeter Wave, edited by Howard A. MacEwen, Giovanni G. Fazio, Makenzie Lystrup, Proc. of SPIE Vol. 9904, 99046D · ( 2016 SPIE · CCC code: 0277-786X/16/\$18 · doi: 10.1117/12.2231736 
Delivery of diffraction limited science images is one of the key drivers for doing optical/nearIR astronomy from space, so it is paramount that the optical elements are properly aligned. The act of co-phasing is the removal of rigid-body alignment errors between primary mirror segments such that they deliver the same resolution as a monolithic primary would produce. There are several mature and effective methods for measuring phase aberrations in optical systems; these include phase retrieval, famously used to determine the correction factor to be applied by COSTAR, the corrective optical package installed on the Hubble Space Telescope, ${ }^{6}$ other similar methods include prescription retrieval. ${ }^{7}$ The Keck telescopes show the successful co-phasing of a large segmented aperture using modified SH WFS and curvature WFSing, ${ }^{8,9}$ serving as the pathfinder for the JWST Dispersed Fringe Sensor. ${ }^{10}$ The phase retrieval and related methods employ high computational complexity; often a multi-parameter merit function is minimized, requiring multiple Fourier transforms for each iteration, and a high-precision mechanized element is required to translate the sensor through the image plane by a known amount with very little run-out. The Dispersed Fringe Sensor is reliant on dispersive optics and requires its own additional optical

path. We propose a method which can easily be inserted into an optical system from a physical perspective, and which executes significantly less complex algorithms to determine the phase errors, making it interesting in general and useful specifically in the case of cube-sat sized/powered CPU technology, which has limited ability to execute large numbers of FFTs in a reasonable time-frame.

\subsection{Phase Contrast}

Phase shifting the DC component of the focal plane PSF leads to constructive or destructive interference between phase shifted and un-phase shifted portions of the wavefronts, leading to changes in the amplitude of the electric field. It can be shown that the intensity, given by $I=E E^{*}$, at the pupil conjugate plane is a function of the phase at the pupil stop and the amount of phase shift introduced at the focal plane. The electric field of a uniform amplitude wavefront at the input pupil of a system is given by

$$
E(u, v)=P(u, v) A(1+\varepsilon(u, v)) e^{i \phi(u, v)}
$$

where $(u, v)$ are pupil-plane coordinates, $P$ is the pupil function, $A$ is the mean electric field amplitude, $\varepsilon$ is the variation in field amplitude over the pupil due to non-uniform illumination, and $\phi$ is the pupil-dependent phase. if $\phi(u, v)$ is small compared to a radian, it can be approximated by a first order Taylor expansion,

$$
E(u, v) \approx P(u, v) A(1+\varepsilon(u, v)+i \phi(u, v))
$$

The electric field is propagated to the focal plane by taking the Fourier Transform,

$$
E(\eta, \nu)=\mathfrak{F}[P(u, v)] * \mathfrak{F}[A(1+\varepsilon(u, v)+i \phi(u, v))],
$$

which can be expanded as,

$$
\begin{gathered}
E(\eta, \nu) \quad A \mathfrak{F}[P(u, v)] \\
+A \mathfrak{F}[P(u, v)] * \mathfrak{F}[\varepsilon(u, v)+i \phi(u, v)]
\end{gathered}
$$

The first term in the right-hand side of Eq 4 is the "DC" component of the electric field. Introducing a phase shift, $\theta$ to the DC component at the focal plane, and taking the inverse 
Fourier Transform to propagate the electric field to the output pupil, gives the expression for the phase shifted electric field,

$$
E(x, y)=A P(x, y)\left(e^{i \theta}+\varepsilon(x, y)+i \phi(x, y)\right) .
$$

To express intensity at the output pupil, take the square modulus, $E E^{*}$, where ${ }^{*}$ represents the complex conjugate, and re-write the complex exponentials using Euler's equation; also drop coordinate signifiers $x, y$ and assume A is defined by pupil function $P(x, y)$, as all subsequent expressions refer to the output pupil plane,

$$
\begin{aligned}
& I=\quad A^{2}\left(e^{i \theta}+\varepsilon+i \phi\right)\left(e^{-i \theta}+\varepsilon-i \phi\right) \\
& I=A^{2}\left(1+2 \varepsilon \cos \theta+2 \phi \sin \theta+\varepsilon^{2}+\phi^{2}\right)
\end{aligned}
$$

It can be seen from Eq. 7 that this intensity function oscillates as a function of $\theta$ with a period of $2 \pi$.

\subsection{White Light Fringes}

The periodic intensity modulation outlined in the previous section is wavelength dependent; thus, for a fixed physical path difference within the Zernike phase plate, an optical path difference (OPD) at each wavelength $\lambda_{i}$ can be computed. Assuming, for now, that all E-field propagation is in air, the $\mathrm{E}$ field at the central wavelength $\lambda_{0}$ is given as,

$$
E_{\lambda_{0}}=A e^{2 \pi i \phi_{0} / \lambda_{0}},
$$

where $\phi_{0}$ is a fixed physical distance. This corresponds to a phase, $\theta$ in radians,

$$
\frac{2 \pi \phi_{0}}{\lambda_{0}}=\theta_{0},
$$

leading to an expression for the $\phi_{0}$ in terms of wavelength and phase:

$$
\phi_{0}=\frac{\theta_{0} \lambda_{0}}{2 \pi} .
$$

For any fixed physical path difference, $\phi_{0}$, we can use Eq 10 to derive the the wavelength dependant phase shift, $\theta_{i}$,

$$
\begin{gathered}
\frac{2 \pi \phi_{0}}{\lambda_{i}}=\theta_{i} \\
\frac{2 \pi \theta_{0} \lambda_{0}}{2 \pi \lambda_{i}}=\theta_{i} \\
\frac{\theta_{0} \lambda_{0}}{\lambda_{i}}=\theta_{i}
\end{gathered}
$$

As the physical path difference is changed, each wavelength modulates at it's own frequency, resulting in a wavelength-dependant intensity modulation period in the pupil plane. For each wavelength, peak intensity occurs when the induced phase shift at the focal plane matches the piston error at the input pupil, and repeats at intervals of $2 \pi$ radians. Summing the intensity modulations of the individual wavelengths or, analogously, using a white light source, creates an 
Table 1: Description of fit parameters of white light fringe equation

\begin{tabular}{c|l} 
Param & Description \\
\hline \hline $\mathrm{a}$ & DC offset or mean intensity \\
$\mathrm{m}$ & fringe slope \\
$\mathrm{c}$ & width of fringe envelope $\left(\approx \Delta \lambda / \lambda_{0}\right)$ \\
$\mathrm{d}$ & unit step size (in $\mu m)$ \\
$\mathrm{e}$ & centre of white-light fringe envelope \\
$\mathrm{A}$ & magnitude of cosine component \\
$\Phi$ & magnitude of sine component
\end{tabular}

intensity fluctuation at the output pupil as a function of focal plane phase shift which exhibits a beat frequency, or fringe pattern, where the peak of the beat envelope corresponds to the piston error at the input pupil. The location of this peak can be found by fitting the measured white light intensity modulations to the following 7 parameter theoretical fringe equation:

$$
F=a+m(x-e)+\frac{\sin \left[\pi d(x+e) \frac{c}{\lambda_{0}}\right]}{\pi d(x+e) \frac{c}{\lambda_{0}}}\left(A \cos \left[d \frac{2 \pi}{\lambda_{0}}(x-e)\right]+\Phi \sin \left[d \frac{2 \pi}{\lambda_{0}}(x-e)\right]\right) .
$$

This equation assumes a constant distribution of wavelengths $\lambda_{\min }<\lambda_{i}<\lambda_{\max }$ centred around $\lambda_{0}$; it can be modified for other types of input such as gaussian or blackbody distributions. The physical meaning of the fitting parameters are described in Table 1.

\subsection{Fine Phasing}

Fine phasing, restricted to phase errors less than $\pi / 2$, with the Dynamic ZWFS has previously been proposed. ${ }^{11}$ The equation of the phase shifted electric field given in 5 is given for four special phase shift cases: $\theta=[-\pi / 2,0, \pi / 2,-\pi]$. In these cases, Eq. 5 reduces to the following:

$$
\begin{aligned}
& E_{1}=A(-i+\varepsilon+i \phi) \\
& E_{2}=A(1+\varepsilon+i \phi) \\
& E_{3}=A(i+\varepsilon+i \phi) \\
& E_{4}=A(-1+\varepsilon+i \phi),
\end{aligned}
$$

and the pupil plane intensity in Eq. 7 for each case reduces to:

$$
\begin{aligned}
& I_{1}=A^{2}\left(1+\varepsilon^{2}-2 \phi+\phi^{2}\right) \\
& I_{2}=A^{2}\left(1+\varepsilon^{2}+2 \epsilon+\phi^{2}\right) \\
& I_{3}=A^{2}\left(1+\varepsilon^{2}+2 \phi+\phi^{2}\right) \\
& I_{4}=A^{2}\left(1+\varepsilon^{2}-2 \epsilon+\phi^{2}\right) .
\end{aligned}
$$

From these four measurements, it is shown in ${ }^{11}$ that the phase error at all spatial coordinates in the pupil can be computed as, 


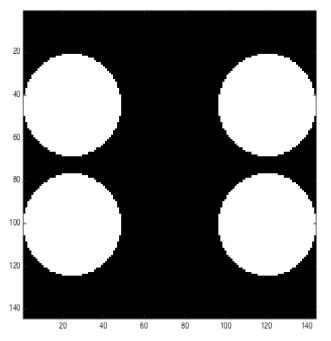

(a)

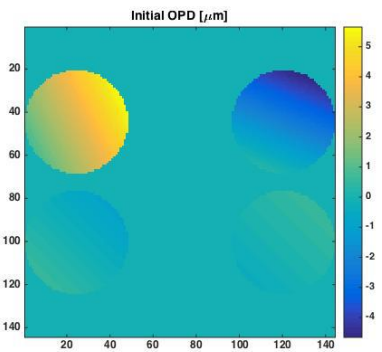

(b)

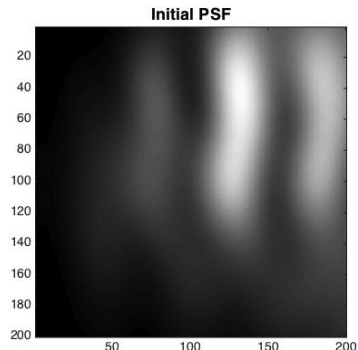

(c)

Figure 1: (a) Pupil mask used in simulations. (b) Initial optical path error $+/-10$ waves. (c) Initial broadband PSF resulting from the pupil in (a) and the phase error in (b).

$$
\phi=\frac{I_{3}-I_{1}}{4 I_{0}} .
$$

The amplitude error term can also be extracted from,

$$
\varepsilon=\frac{I_{2}-I_{4}}{4 I_{0}}
$$

$I_{0}$ is a normalization term.

These are very convenient relationships, however very precise phase shifts are required to achieve this special case and manufacturing errors in the optical phase shifting element are of particular concern for practical applications. Fortunately a general solution exists; it can be shown that both $\phi$ and $\varepsilon$ can be extracted from four images with arbitrary phase shifts, $\theta=\left[\theta_{1}, \theta_{2}, \theta_{3}, \theta_{4}\right]$ :

$$
\phi=\frac{\left(I_{3}-I_{1}\right)\left(\cos \theta_{4}-\cos \theta_{2}\right)-\left(I_{4}-I_{2}\right)\left(\cos \theta_{3}-\cos \theta_{1}\right)}{2 I_{0}\left[\left(\sin \theta_{3}-\sin \theta_{1}\right)\left(\cos \theta_{4}-\cos \theta_{2}\right)-\left(\sin \theta_{4}-\sin \theta_{2}\right)\left(\cos \theta_{3}-\cos \theta_{1}\right)\right]},
$$

and this reduces to Eq. 23 for the special case mentioned above.

\section{SIMULATION RESULTS}

The simulations were carried out in Matlab and implement the efficient Fourier Transform technique described by Soummer ${ }^{12}$ for fast processing, and code elements from the Object Oriented Matlab Adaptive Optics (OOMAO) toolbox for SH-WFS simulation elements. ${ }^{13}$

The simulations were executed using the segmented pupil representing the AAReST ${ }^{14,15}$ segmented primary mirror, which is shown in Fig. 1(a), but will work equally on any form of input pupil, segmented or not and independent of segment gap size.

One example of an initial pupil condition can be seen in Figs 1(b) and 1(c), which show the phase error in the pupil and the resulting PSF respectively. Applying a phase shift excursion of $+/-12$ waves, and recording the pupil conjugate plane intensity of each segment at each step provides the white light fringe measurement data required for the coarse phasing step using Eq. 14. The results for a single segment are shown in Fig. 2; the fit goodness is $97 \%$ using a phase step resolution of $180 \mathrm{~nm}$ per step, a bandwidth of $240 \mathrm{~nm}$ and $20 \mathrm{~nm}$ wavelength step resolution over the bandpass to simulate the white light PSF. 


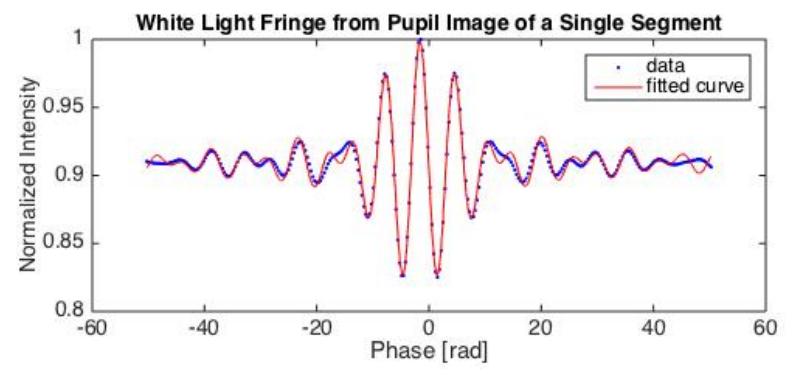

Figure 2: Coarse phasing white light fringe pattern from simulated measurement data, and the resulting fitted curve using Eq. 14.

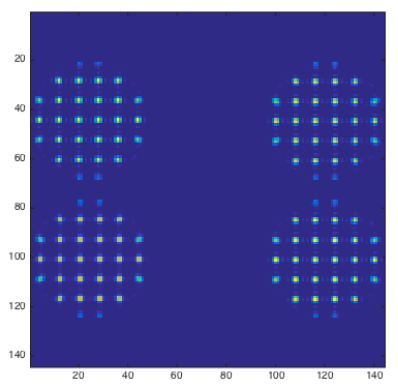

(a)

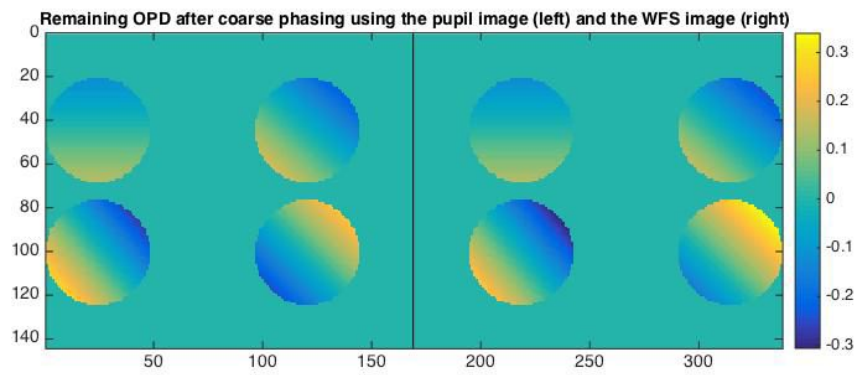

(b)

Figure 3: (a) Pupil image sampled by SH WFS. (b) Residual OPD after coarse phasing step. Left: Pupil Image result. Right: WFS image result.

\subsection{Shack-Hartmann WFS for pupil imaging}

The fine phasing step has previously been shown to work in simulation. ${ }^{11}$ As an extension of this result, we investigated the impact of using SH-WFS spots rather than a full pupil image (Fig. $3(\mathrm{a})$ ) to collect the measurements for fringe fitting. A comparison between the accuracy of phase error measurements using full pupil images and using SH-WFS image was made in simulation for both coarse and fine phasing steps. The resulting residual phase error after coarse phasing using both methods are shown in Fig. 3(b), and the final PSFs after correcting identical initial phase errors are compared in Fig. 4. Small differences in results are due to signal averaging in the WFS measurement; in this case, each WFS spot is $2 \times 2$ pixels and the lenslets sample 

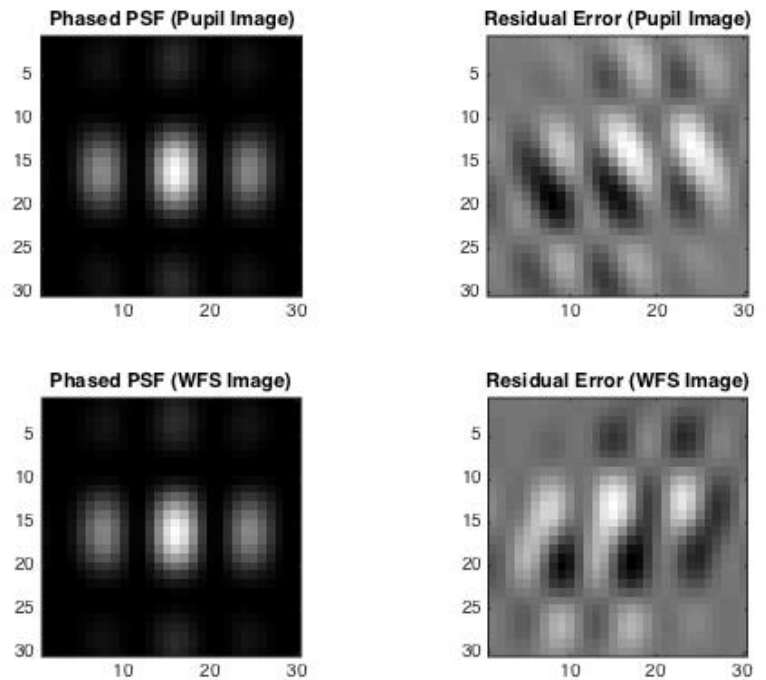

Figure 4: Residual PSF after phasing. Top row: Pupil Image result. Bottom row: WFS Image result.
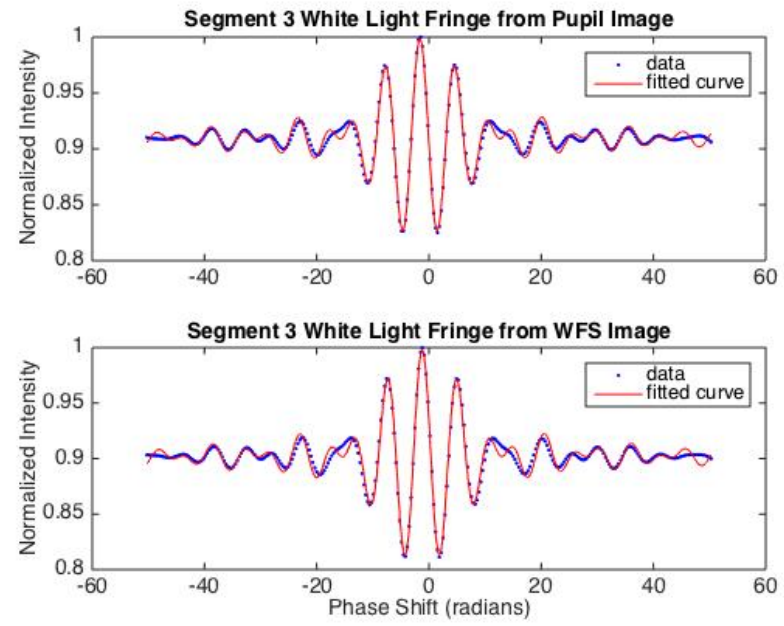

Figure 5: The fitting of the white light fringe equation is unaffected by the source of the measurement data.

the pupil at a resolution of $8 \times 8$ pixels. Additionally, in the command reconstruction step the measured phase error is projected onto the Zernike modes 2 and 3 (tip and tilt). The differing resolution of the two types of measurements - number of pixels in the case of the pupil image and number of lenslets in the case of the WFS image - leads to small numerical differences in the projection. Both methods have more than enough resolution to accurately estimate such low orders as tip/tilt, so the overall final result is minimally affected. Both methods deliver a final phased pupil with an RMS error of less than $\lambda / 80$. These results show that the two methods are indeed equivalent so long as appropriate care is taken during image processing. 


\section{ZERNIKE PHASE PLATE DESIGN}

The manufacturing concept for the long stroke ZWFS is shown in Fig. 6. It incorporates a large number of individual OPD steps both positive and negative in a slot shaped staircase. The width of the slot is nominally equal to the radius of the Airy disk of the phased primary, but simulations show that the phase plate is just as effective for up to several times this width. The length of each tread of the staircase is $0.5 \mathrm{~mm}$ which is a value selected such that there is minimal interference from adjacent steps, meaning there should not be any phase shift induced on the wings of the PSF from other steps. The step height is chosen such that the change in OPD from one step to the next is approximately $\pi / 2$ at the central wavelength. The exact value of the height will change depending on the index of refraction of the material (for a transmissive phase plate) and the central wavelength. Fabrication of such a device is in progress using electron beam lithography. Figure 7 shows the resist pattern to be etched into a fused silica wafer. The dimensions are exaggerated for display, the actual width of the staircase is $50 \mu m$, the excursion from the reference plane is approximately $+/-1 \mu \mathrm{m}$, and the width of the reference plane (blue area) is approximately $1 \mathrm{~mm}$. Only the inner steps are shown, the entire device will have 40 steps.
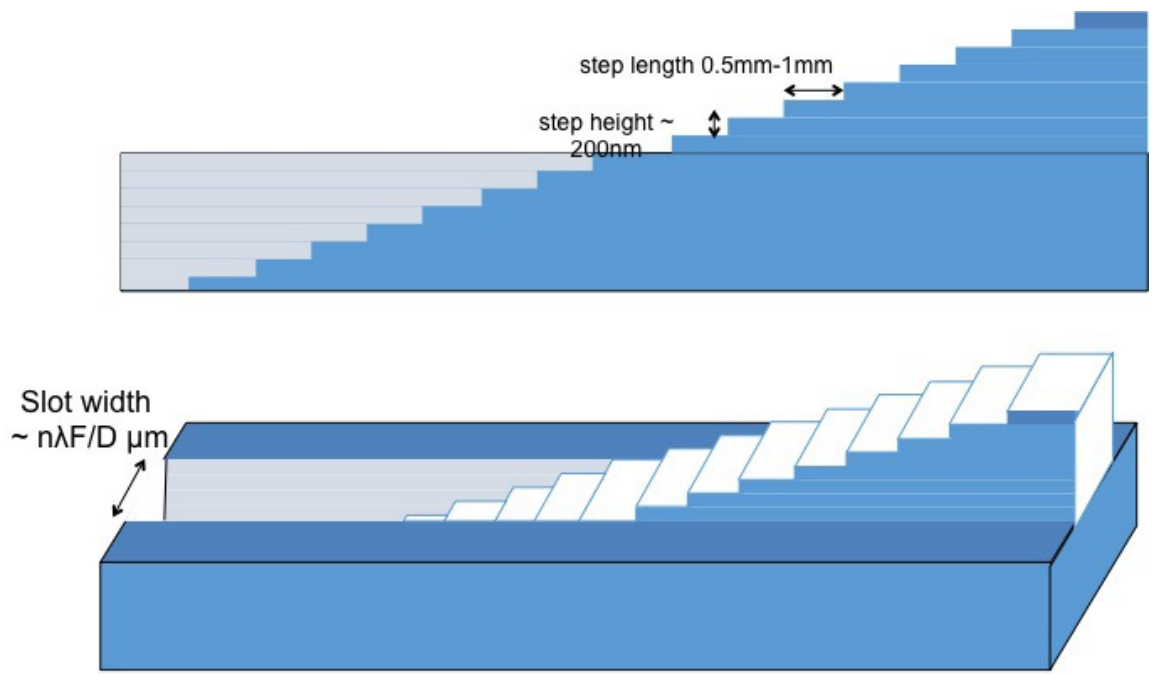

Figure 6: Concept for achieving the extended dynamic range of the long stroke ZWFS (not to scale). The original centro-symmetric dimple has been replaced with a slot; within the slot are varying step heights both negative and positive relative to the base plane. Top: side view. Bottom: parametric view.

\section{CONCLUSIONS AND FUTURE WORK}

A high dynamic range method of co-phasing segmented apertures has been proposed, and the theory, performance and conceptual design is outlined. A simulation tool has been built which focusses on the realistic implementation of a long stroke ZWFS for large capture range piston errors, limited only by the maximum OPD built into the Zernike phase plate. It has shown that phasing can be carried out successfully, even when very loose constraints are placed on the shape and size of the phase shifting element. We have shown that, so long as the particular phase shifts are known - i.e. the phase plate is well characterized - both coarse and fine phasing are reliable for a variety of step resolutions both uniform and non uniform. The tool also shows that phasing can be carried out using a SHWFS as the pupil imager, making it very easy to integrate optically 


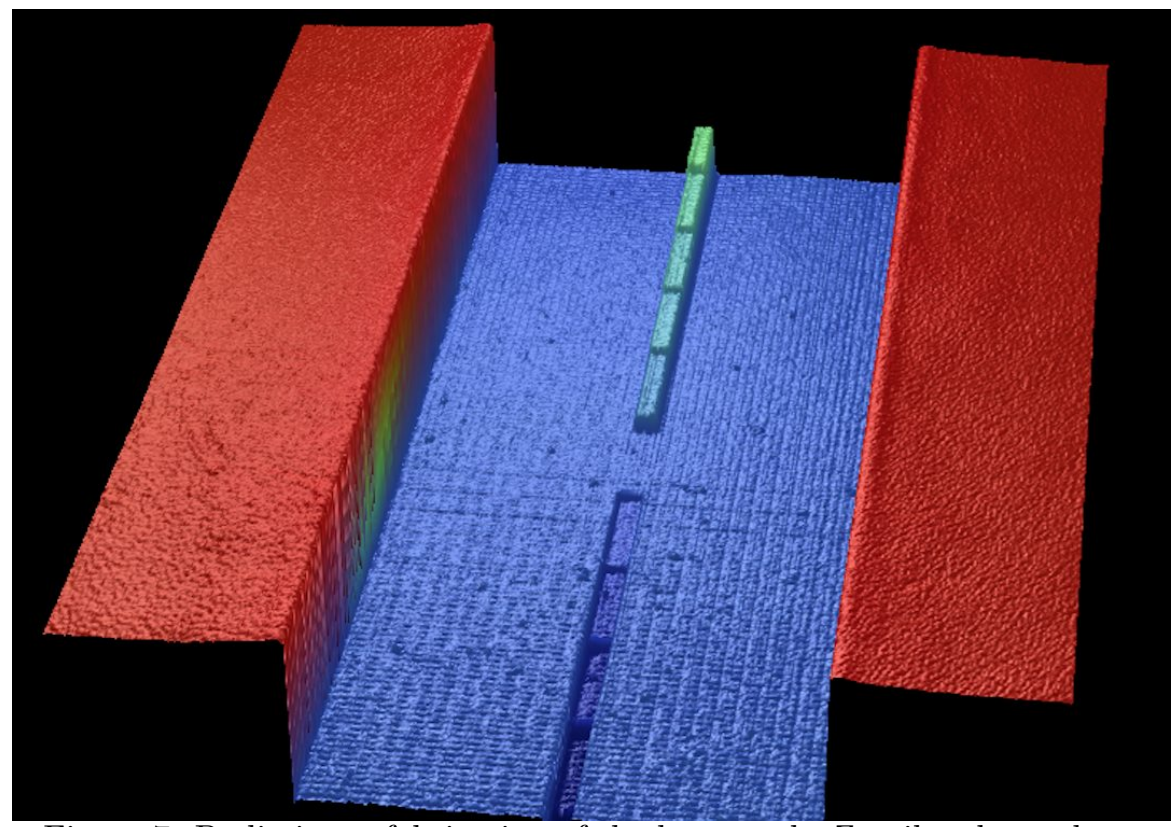

Figure 7: Preliminary fabrication of the long-stroke Zernike phase plate.

into an existing AO system. A test Zernike phase plate has been designed and future work will include the completion of its manufacture and incorporation into a segmented telescope testbed, the work on-which is currently on-going.

\section{ACKNOWLEDGEMENTS}

This work was funded in part by the Spontaneous Research and Technology Development fund at the Jet Propulsion Laboratory. This work was partially carried out by the Jet Propulsion Laboratory, California Institute of Technology, under a contract with the National Aeronautics and Space Administration. 


\section{REFERENCES}

[1] Sanders, G. H., "The thirty meter telescope (tmt): An international observatory," Journal of Astrophysics and Astronomy 34(2), 81-86 (2013).

[2] Shectman, S. and Johns, M., "Gmt overview," in [SPIE Astronomical Telescopes+ Instrumentation], 77331Y-77331Y, International Society for Optics and Photonics (2010).

[3] Gilmozzi, R. and Spyromilio, J., "The european extremely large telescope (e-elt)," The Messenger 127(11), 3 (2007).

[4] Stahl, H. P., "Jwst mirror technology development results," in [Optical Engineering+ Applications], 667102-667102, International Society for Optics and Photonics (2007).

[5] Postman, M., Argabright, V., Arnold, B., Aronstein, D., Atcheson, P., Blouke, M., Brown, T., Calzetti, D., Cash, W., Clampin, M., et al., "Advanced technology large-aperture space telescope (atlast): A technology roadmap for the next decade," arXiv preprint arXiv:0904.0941 (2009).

[6] Fienup, J. R., Marron, J. C., Schulz, T. J., and Seldin, J. H., "Hubble space telescope characterized by using phase-retrieval algorithms," Applied optics 32(10), 1747-1767 (1993).

[7] Redding, D., Dumont, P., and Yu, J., "Hubble space telescope prescription retrieval," Applied optics 32(10), 1728-1736 (1993).

[8] Chanan, G., Troy, M., Dekens, F., Michaels, S., Nelson, J., Mast, T., and Kirkman, D., "Phasing the mirror segments of the keck telescopes: the broadband phasing algorithm," Applied Optics 37(1), 140-155 (1998).

[9] Chanan, G., Troy, M., and Sirko, E., "Phase discontinuity sensing: a method for phasing segmented mirrors in the infrared," Applied optics 38(4), 704-713 (1999).

[10] Zhang, Y., Liu, G.-R., Wang, Y.-F., Li, Y.-P., Zhang, Y.-J., and Zhang, L., "Preliminary study of a dispersed fringe type sensing system," Research in Astronomy and Astrophysics 9(8), 945 (2009).

[11] Wallace, J. K., Rao, S., Jensen-Clem, R. M., and Serabyn, G., "Phase-shifting zernike interferometer wavefront sensor," Proc. SPIE 8126, 81260F-81260F-11 (2011).

[12] Soummer, R., Pueyo, L., Sivaramakrishnan, A., and Vanderbei, R. J., "Fast computation of lyot-style coronagraph propagation," Opt. Express 15, 15935-15951 (Nov 2007).

[13] Conan, R. and Correia, C., "Object-oriented matlab adaptive optics toolbox," Proc. SPIE 9148, 91486C-91486C-17 (2014).

[14] Underwood, C. and Pellegrino, S., "Autonomous assembly of a reconfigurable space telescope (aarest) for astronomy and earth observation," in [8th IAA Symposium on Small Satellites for Earth Observation, Berlin], 4-8 (2011).

[15] Underwood, C., Pellegrino, S., Lappas, V., Bridges, C., Taylor, B., Chhaniyara, S., Theodorou, T., Shaw, P., Arya, M., Breckinridge, J., et al., "Autonomous assembly of a reconfiguarble space telescope (aarest)-a cubesat/microsatellite based technology demonstrator," (2013). 